\title{
REACH: Solutions for Technology-Based Prevention and Empowerment for Older People and their Caregivers
}

\author{
Thomas Linner ${ }^{1} \cdot$ Anja Seeliger $^{1} \cdot$ Lydia Vogt $^{1} \cdot$ Barbara Schäpers ${ }^{1}$. \\ Martina Steinböck ${ }^{1} \cdot$ Carmen Krewer $^{1} \cdot$ Thomas Bock $^{1}$
}

Published online: 11 March 2020

(C) The Author(s) 2020

\begin{abstract}
REACH stands for "Responsive Engagement of the Elderly Promoting Activity and Customized Healthcare". Sustained physical activity matters greatly to the health and well-being of older people and significantly improves their chance of maintaining independent living. It can make a difference across the whole care continuum as well as in almost every setting. Therefore, REACH solutions focus on the systematic, targetoriented increase of physical activity of older people, and tackle the whole prevention spectrum (primary, secondary, and tertiary). It seeks to empower older people and their formal and informal caregivers, and works towards viable solutions for both the formal and in-formal care sector. Technology-based personalization of prevention, activation, and care services provided in various living and care settings is at the center of the developed solutions. Ideally toolkit approach would allow for the tailoring of solutions that create value for end-users, care providers and health care payers alike through the combination, integration and adaptation/re-design elements towards the different contexts of different countries, different payment and reimbursement structures. This Special Issue sheds light on such solutions, their conception, their development, and their testing.
\end{abstract}

\section{Background}

According to WHO (World Health Organization 2015, accessed on 15/02/19), people over the age of 65 who engage in a high level of physical activity experience a lower overall mortality, while at the same time improve functional health. It has been found that physical activity in older people has a positive effect, on not only their cognitive and physical function, but it also helps to reduce stress and therefore promote a higher

Thomas Linner

thomas.linner@br2.ar.tum.de

Technical University of Munich, Munich, Germany 
quality of life (Awick et al. 2017). Evidence has shown that a lack of physical activity is a major risk factor for a higher rate in mortality, disease and disability (Physical Activity Guidelines 2018). Basic activities of daily living - such as getting up safely from a chair and sitting down safely - are preconditions for maintaining autonomy in people aged 65 and above. Difficulties and unexpected mishaps during these activities can lead to an inactive lifestyle, resulting in a decline in their physical condition. A fall during mobility-related activities can be caused by different influences (Zijlstra et al. 2012). For example, an older person has more difficulty anticipating or reacting to unexpected forces due to general age-related deterioration in neuromuscular and vestibular functioning in addition to orthostatic intolerance.

Furthermore, falls are a common problem in the older population leading to a loss of independence (Blake et al. 1988). One third of the population over the age of 65 has at least one serious fall with negative effects per year (Poziac et al. 2016). Of all falls, $41 \%$ are attributed to a mistake during a position change (e.g. standing to sitting) (Rapp et al. 2012). In general, falls among older people cause injuries. In the health system of the USA, these incidents amount to $\$ 34$ billion per year. Those fall-related costs could be significantly reduced by using preventive training strategies (Poziac et al. 2016). Furthermore, balance and strength training, orthopaedic shoe modification and an individualised drug management can reduce the number of falls significantly (Poziac et al. 2016). Overall, evidence shows that, among older persons in particular, regular physical activity reduces the risk of frailty, falls, dementia, severe injuries after falls, and other major cardiovascular or metabolic health risks (Physical Activity Guidelines 2018). Regular, moderate physical activity can maintain muscle strength and considerably improve bone density (Scott et al. 2019). In addition, it is recommended to avoid polypharmacy, and to induce nutrition changes such as an intake of adequate calcium (Province et al. 1995). Through early detection of physical inactivity and personalised early intervention, the REACH system aims to engage older individuals in continued activity and thus prevent deterioration in their health.

Specifically, REACH-administered systematic, technology-based and communityintegrated physical activation enables:

a) an increase of functional ability (in terms of ADLs, mobility, balance, walking speed, grip strength, etc.);

b) an increase of cardiac health and a reduction of risk of chronic diseases (e.g. cardiovascular diseases, diabetes, etc.) and mortality;

c) cognitive fitness and social participation (self-efficacy, less loneliness, etc.);

d) general resilience (e.g. ability to regain health after hospital admission) and safety

Sustained physical activity matters greatly to the health and well-being of older people and significantly improves their chance of maintaining independent living. Moreover, it can make a difference across the whole care continuum (early/later stages, levels and types of care, etc.) as well as in almost every community or use case setting for the older population in Europe. Through this broad impact, REACH contributes in a proactive and preventative manner to both a) a healthier and more independent elderly population in Europe and b) a reduction of health care and social costs associated with an ageing population. 
In this context, REACH creates new market opportunities for the European industry, including SMEs to capitalize on European high-tech-knowhow, to make Europe a market leader in prevention and empowerment technologies, meanwhile tackling the ultimate cause of rising healthcare expenditures. Therefore, REACH focuses on the systematic, target-oriented increase of physical activity of older people, and tackles in this context the whole prevention spectrum (primary, secondary, and tertiary). It seeks to empower older people and their formal and informal caregivers, and works towards viable solutions for both the formal and in-formal care sector. Technology-based personalization of prevention, activation, and care services provided in various living and care settings is at the center of the developed solutions.

\section{The REACH Framework}

REACH - as a sensing-monitoring-intervention system - enables, on the one hand, early detection of critical events and critical changes in the behaviour of older people (short-term and long-term changes). On the other hand, it improves physical and functional ability of older people, and strengthens their autonomy and capacity for independent living by motivating them to improve and maintain physical and socio-cognitive status and by supporting a healthier lifestyle. REACH situates Touchpoints according to the nature of the analytical models they incorporate in Garter's categorisation graph (Fig. 1). A toolkit guides the technical implementation of REACH. The toolkit comprises a series of partially independent components or "raw elements", which can be classified into 11 categories: sensors, analytics and Machine Learning tools, devices, smart furniture, exercise and behaviour change schematics, human-machine-interfaces, data storage platforms etc. REACH has developed and refined a design methodology (Sensing-Monitoring-Intervention/SMI workflow) for the use-case-specific combination and integration of these elements.

The REACH toolkit approach allows for the tailoring of solutions that create value for end-users, care providers and health care payers alike. It does so through the

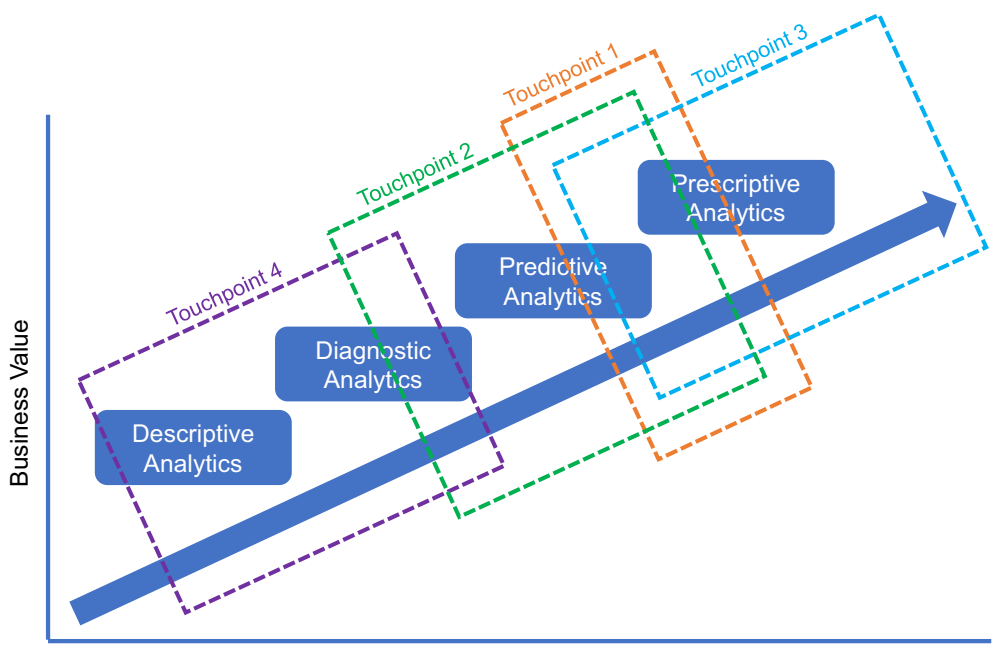

Data Analytics Complexity

Fig. 1 Analytical models used by the REACH sub-systems (based on Gartner's categorisation graph) 


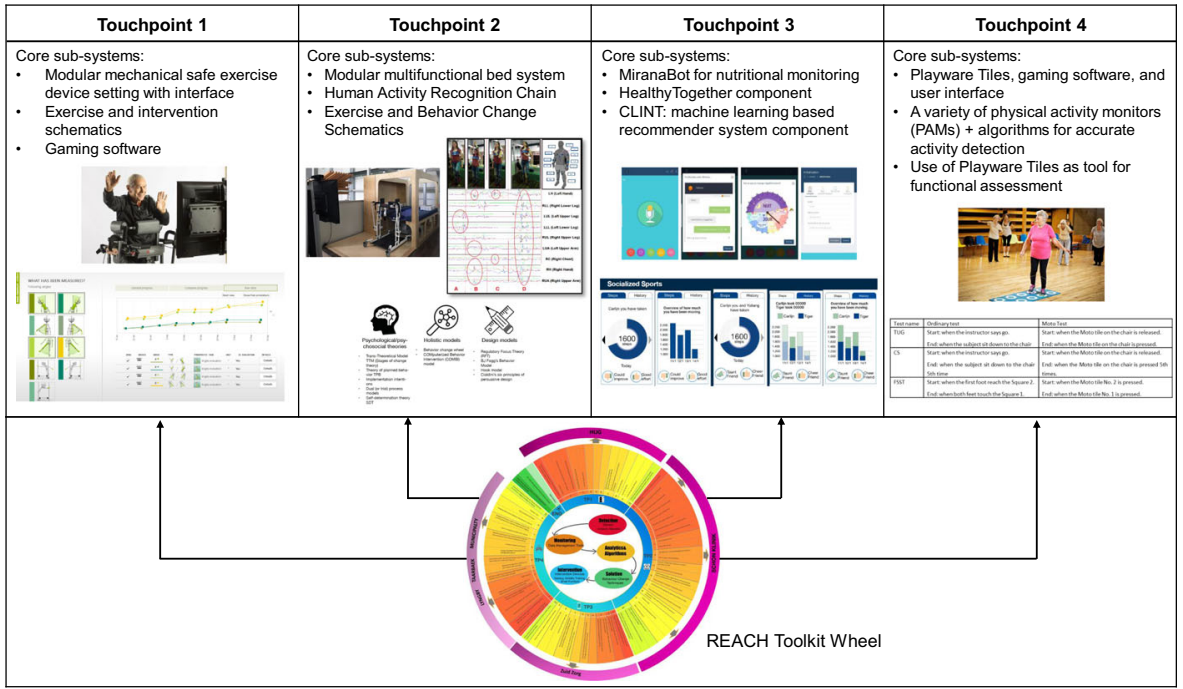

Fig. 2 REACH Toolkit Wheel and its connection to the four Touchpoints

combination, integration and adaptation/re-design of its elements towards the different contexts of different countries, different payment and reimbursement structures (e.g., insurance or tax-based), specific use case settings and processes and, most importantly, individual end-user needs and capabilities. (SK/Schön Klinik, HUG/Geneva Hospital, ZZ/ZuidZorg, Lyngby/Lyngby Municipality). In this context, REACH demonstrates its ability to integrate, cross-integrate, share and interchange its elements and co-create new case-specific elements for each use case setting (see Fig. 2).

\section{Ecosystem Approach and System Verification and Validation by Trials}

REACH achieves its objectives through highly integrated sensing-monitoring-intervention chains representing comprehensive solutions that are exemplarily and iteratively adapted in the project to the ecosystems of a series of care settings throughout Europe (homes, hospitals, care homes, day care facilities, communities, etc.) for older individuals. REACH implements, demonstrates, tests, and validates (by more than 27 small- to medium-sized trials) through those settings, customized and personalized instances of this chain. A unique feature of REACH is the integrated utilization of personalized behavior change and engagement techniques informing about the development of the products and solutions (sensors, interfaces, devices, etc.). REACH implements a combination of wearable and ambient sensors for each Touchpoint along with a set of co-adapted Machine Learning elements. Machine Learning is used as a core element in multiple ways, e.g. to predict Activities of Daily Living (ADLs), recognize physical activity and behavioural trends, detect deviations of patterns and critical situations, cluster and profile people, and inform the effectiveness of the assignment of certain interventions. Personalised Intelligent Interior Units ( $\mathrm{PI}^{2} \mathrm{Us}$; smart furniture devices) are used to seamlessly integrate the aforementioned functional elements into daily life in the different target use case settings. Lastly, but equally important, REACH has developed practices and schematics to assess the implications of the use of its solutions with regard to privacy, legal, and ethical aspects in order to ensure 


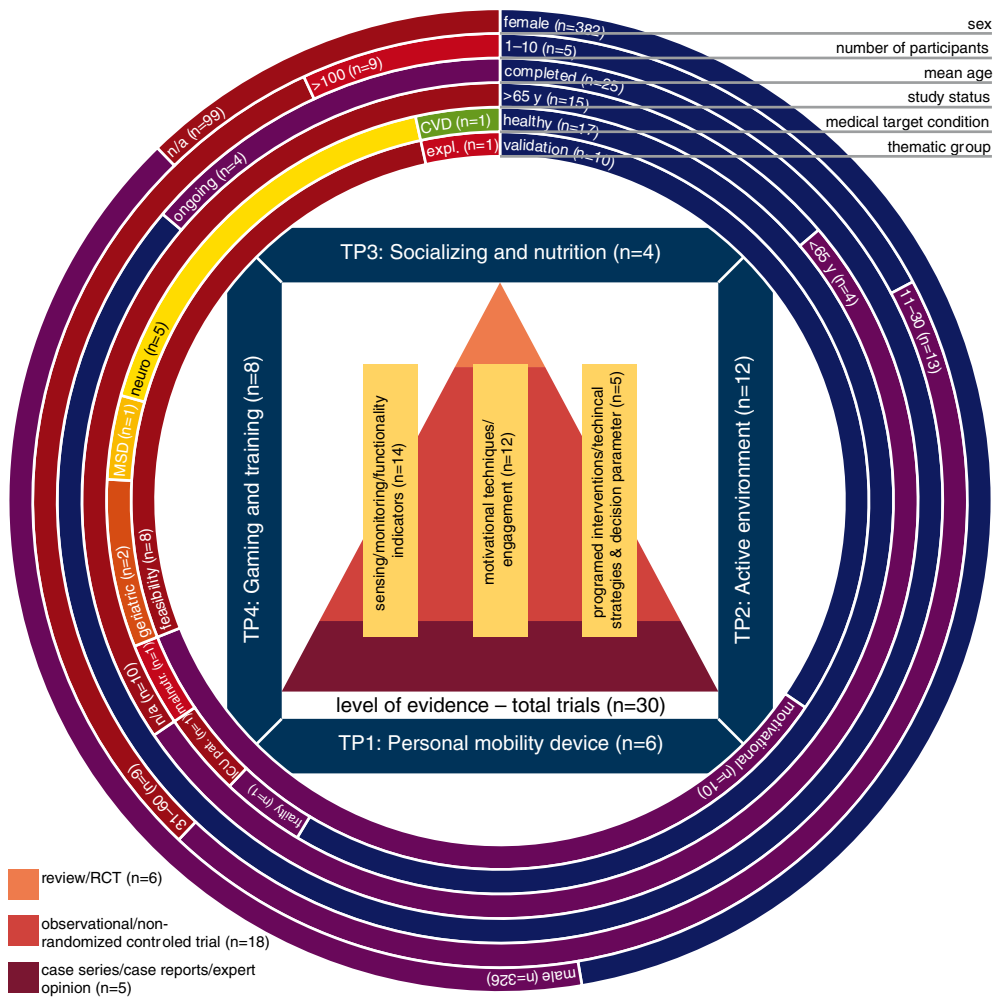

Fig. 3 Overview of trials conducted in REACH (Image: C. Krewer, M. Schlandt)

technology acceptance by end-users, caregivers, and other care and medical professionals (see Fig. 3.).

\section{Standardization in the REACH Context}

Standardization is a key strategic tool in order to agree on terminologies, methodologies, requirements, quality criteria etc. in a specific field to make a product, process or service fit for its purpose (EN 45020: 2006). Thereby, standardization can drive innovations. Seen from the perspective of the already existing standardization landscape, active and healthy ageing touches a variety of different and very broad fields, such as Active and Assisted Living (AAL), accessibility, health services, assistive products, design for all, etc. TCs within the international, European and national standardization bodies cover these topics. REACH synthesised existing and ongoing standardisation activities and got involved in the formation of a new ISO TC (e.g. ISO TC 314 Ageing Societies) focusing on standardisation of the community context of care innovations, contributed to technical standardisation (e.g. IEC SyC Active Assisted Living), and made first steps towards standardisation of risk governance mechanisms in the context of the deployment of sensing an monitoring technology (CEN CWA "Guidelines for introducing ambient and wearable monitoring technologies balancing privacy protection against the need for oversight and care"). 


\section{Conclusion and Overview of the Articles Featured in this Special Issue}

This Special Issue sheds light on key elements developed by REACH for future active ageing solutions that were introduced in the previous sections. Foremost, the integrated development of sensing systems (consisting of wearable and ambient sensor), Human Activity Recognition chains, and the associated machine learning models are key to get solid insights into the activities and behaviours of older people (Schrader et al. 2020). Based on such approaches, recommendation systems can be built, which profile users and calculate and facilitate the optimal assignment, design, and personalisation of possible interventions (Kulev et al. 2020) Based on this, effective interventions through devices for physical activation (target-oriented training of balance, muscle strength, functional abilities, cardiac health, etc.) and digitally enabled and gamified training programs can be administered and integrated seamlessly into care settings (Randriambelonoro et al. 2020). Strategies and techniques from the field of behaviour change and motivation can be seamlessly integrated into these solutions to improve aspects such as adherence, engagement, and health outcomes (Ehrari et al. 2020). Similarly, approaches of nutritional monitoring and interventions complement approaches to physical and functional empowerment (Rusu et al. 2020). Lastly, but equally important, smart furniture elements serve as carrier elements that allow for an easy and fast deployment of advanced multi-component solutions (Hu et al. 2020).

Acknowledgements Open Access funding provided by Projekt DEAL. REACH has received funding from European Union's Horizon 2020 research and innovation programme under grant agreement No 690425. REACH stands for "Responsive Engagement of the Elderly Promoting Activity and Customized Healthcare". The proposal for this project was developed in 2015 and submitted under Pillar 3 of H2020 in Societal Challenge 1 Personalized Healthcare (PHC). In the European consortium with 17 partners from higher education institutions and industry, the four EuroTech Universities along with the industry partners (including leading European health care technology, rehabilitation, and care and hospital firms) build the core of this project. The total grant amounts to just over 6 Million Euros.

Open Access This article is licensed under a Creative Commons Attribution 4.0 International License, which permits use, sharing, adaptation, distribution and reproduction in any medium or format, as long as you give appropriate credit to the original author(s) and the source, provide a link to the Creative Commons licence, and indicate if changes were made. The images or other third party material in this article are included in the article's Creative Commons licence, unless indicated otherwise in a credit line to the material. If material is not included in the article's Creative Commons licence and your intended use is not permitted by statutory regulation or exceeds the permitted use, you will need to obtain permission directly from the copyright holder. To view a copy of this licence, visit http://creativecommons.org/licenses/by/4.0/.

\section{References}

Awick, E. A., Ehlers, D. K., Aguiñaga, S., Daugherty, A. M., Kramer, A. F., \& McAuley, E. (2017). Effects of a randomized exercise trial on physical activity, psychological distress and quality of life in older adults. General Hospital Psychiatry, 49, 44-50.

Blake, A., Morgan, K., Bendall, M. J., Dallosso, H., Eebrahim, S. B., Arie, T. H., Fentem, P. H., \& Bassey, E. J. (1988). Falls by elderly people at home: Prevalence and associated factors. Age and Ageing, 17, 365-372.

EN 45020:2006 Standardization and related activities - General vocabulary (ISO/IEC Guide 2:2004).

ISO TC 314 Ageing Societies, https://committee.iso.org/home/tc314

Physical Activity Guidelines Advisory Committee Scientific Report. (2018). Part D. Integrating the Evidence. https://health.gov/paguidelines/second-edition/report/pdf/05_D_Integrating_the_Evidence.pdf. Accessed 19.02.2019. 
Province, M. A., Hadley, E. C., Hornbrook, M. C., Lipsitz, L. A., Miller, J. P., Mulrow, C. D., Ory, M. G., Sattin, R. W., Tinetti, M. E., \& Wolf, S. L. (1995). The effects of exercise on falls in elderly patients. A preplanned meta-analysis of the FICSIT trials. Frailty and injuries: cooperative studies of intervention techniques. Jama, 273, 1341-1347.

Rapp, K., Becker, C., Cameron, I. D., König, H.-H., Büchele, G. (2012) Epidemiology of falls in residential aged care: Analysis of more than 70,000 falls from residents of Bavarian nursing homes. Journal of the American Medical Directors Association, 13(2), 187.e1-187.e6.

Scott, D., Johannson, J., McMillan, L. B., Ebeling, P. R., Nordstrom, A., \& Nordstrom, P. (2019). Mid-calf skeletal muscle density and its associations with physical activity, bone health and incident 12-month falls in older adults: The healthy ageing initiative. Bone, 120, 446-451.

World Health Organization. (2015): Physical Activity and Older Adults: Recommended levels of physical activity for adults aged 65 and above. http://www.who.int/dietphysicalactivity/factsheet_olderadults/en/. Accessed 15.02.2019.

Zijlstra, A., Mancini, M., Lindemann, U., Chiari, L., Zijlstra, W. (2012). Sit-stand and stand-sit transitions in older adults and patients with Parkinson's disease: Event detection based on motion sensors versus force plates journal of NeuroEngineering and rehabilitation 2012, 9:75.

Publisher's Note Springer Nature remains neutral with regard to jurisdictional claims in published maps and institutional affiliations. 